OPEN ACCESS

Edited by:

Carolina Henritta Pohl, University of the Free State,

South Africa

Reviewed by: Mrudula Patel,

University of the Witwatersrand,

South Africa

Raymond Allan,

De Montfort University,

United Kingdom

*Correspondence:

Geelsu Hwang

geelsuh@upenn.edu

Specialty section:

This article was submitted to

Biofilms,

a section of the journal

Frontiers in Cellular and

Infection Microbiology

Received: 30 October 2020 Accepted: 30 December 2020 Published: 19 February 2021

Citation:

Kim H-E, Liu Y, Dhall A, Bawazir M, Koo H and Hwang G (2021) Synergism of Streptococcus mutans and Candida albicans Reinforces Biofilm Maturation and Acidogenicity in Saliva: An In Vitro Study. Front. Cell. Infect. Microbiol. 10:623980. do: $10.3389 /$ fcimb.2020.623980

\section{Synergism of Streptococcus mutans and Candida albicans Reinforces Biofilm Maturation and Acidogenicity in Saliva: An In Vitro Study}

\author{
Hye-Eun Kim ${ }^{1}$, Yuan Liu ${ }^{1}$, Atul Dhall ${ }^{1}$, Marwa Bawazir ${ }^{1}$, Hyun Koo ${ }^{2,3}$ \\ and Geelsu Hwang ${ }^{1,3 *}$ \\ 1 Department of Preventive and Restorative Sciences, School of Dental Medicine, University of Pennsylvania, Philadelphia, \\ PA, United States, ${ }^{2}$ Department of Orthodontics, School of Dental Medicine, University of Pennsylvania, Philadelphia, PA, \\ United States, ${ }^{3}$ Center for Innovation \& Precision Dentistry, School of Dental Medicine, School of Engineering and Applied \\ Sciences, University of Pennsylvania, Philadelphia, PA, United States
}

Early childhood caries, a virulent-form of dental caries, is painful, difficult, and costly to treat that has been associated with high levels of Streptococcus mutans (Sm) and Candida albicans (Ca) in plaque-biofilms on teeth. These microorganisms appear to develop a symbiotic cross-kingdom interaction that amplifies the virulence of plaquebiofilms. Although biofilm studies reveal synergistic bacterial-fungal association, how these organisms modulate cross-kingdom biofilm formation and enhance its virulence in the presence of saliva remain largely unknown. Here, we compared the properties of Sm and Sm-Ca biofilms cultured in saliva by examining the biofilm structural organization and capability to sustain an acidic $\mathrm{pH}$ environment conducive to enamel demineralization. Intriguingly, Sm-Ca biofilm is rapidly matured and maintained acidic pH-values ( 4.3), while Sm biofilm development was retarded and failed to create an acidic environment when cultured in saliva. In turn, the human enamel slab surface was severely demineralized by Sm-Ca biofilms, while there was minimal damage to the enamel surface by $\mathrm{Sm}$ biofilm. Interestingly, Sm-Ca biofilms exhibited an acidic environment regardless of their hyphal formation ability. Our data reveal the critical role of symbiotic interaction between S. mutans and C. albicans in human saliva in the context of pathogenesis of dental caries, which may explain how the cross-kingdom interaction contributes to enhanced virulence of plaque-biofilm in the oral cavity.

Keywords: cross-kingdom biofilm, Streptococcus mutans, Candida albicans, human saliva, acidogenicity, enamel demineralization

\section{INTRODUCTION}

A hyper-virulent form of tooth decay, Early Childhood Caries (ECC), is a biofilm-derived oral disease induced by protracted ingestion of dietary sugars (Hallett and O'Rourke, 2002; Parisotto et al., 2010; Hajishengallis et al., 2017). Interestingly, opportunistic fungal pathogen Candida albicans is frequently detected with a cariogenic bacterial pathogen Streptococcus mutans in the 
plaque biofilms formed on the tooth surface of children with ECC (Yang et al., 2012; Xiao et al., 2016; Xiao et al., 2018). Previous in vitro and in vivo studies demonstrated the mechanism of bacterial-fungal interaction whereby S. mutansderived exoenzymes, glucosyltransferases B (GtfB), avidly bind to C. albicans and produce extracellular glucans on C. albicans in the presence of sucrose, promoting subsequent $S$. mutans binding to C. albicans (Gregoire et al., 2011; Falsetta et al., 2014; Hwang et al., 2015; Hwang et al., 2017). This enhanced interaction accelerates microbial carriage and production of exopolysaccharides (EPS), facilitating enamel dissolution on the tooth surface due to their acidogenic and aciduric characteristics in a milieu rich in dietary carbohydrates (Hwang et al., 2017; Xiao et al., 2017; Pereira et al., 2018).

Human saliva has been recognized to play a significant role in the homeostasis and symbiosis in complex oral environments, contributing to the balance of health and disease (Dawes and Wong, 2019; Pedersen and Belstrøm, 2019). Particularly, saliva exhibited diverse protective functions against dental caries such as i) facilitating clearing irritants, such as microorganisms and dietary carbohydrates; ii) preventing tooth demineralization via various inorganics (e.g., calcium and phosphorus), buffering acids from dietary carbohydrates and bacterial fermentation byproducts; iii) providing antimicrobial activity through numerous proteins, peptides (e.g., histidine), and antibodies (secretory immunoglobin A) (Hara and Zero, 2014; van't Hof et al., 2014; Colombo et al., 2016; Pedersen and Belstrøm, 2019).

As such, saliva can modulate bacterial adhesion and consequent plaque-biofilm formation directly and indirectly. For instance, salivary agglutinin has been found to alleviate $S$. mutans adherence and biofilm formation (Ahn et al., 2008). In contrast, a pellicle formed on the enamel surface via adsorption of salivary proteins can facilitate bacterial colonization, resulting in the mature oral biofilm (Cheaib et al., 2015). Specifically, oral streptococci exhibited enhanced binding affinity to the salivary glycoproteins such as mucin and $\alpha$-amylase (Murray et al., 1992; Cross and Ruhl, 2018). Interestingly, the adhesion of C. albicans to the surface of oral streptococci (e.g., S. gordonii, S. oralis, and S. sanguinis) was promoted by selective adsorption of basic salivary proline-rich proteins (O'Sullivan et al., 2000). Although biofilm studies reveal prominent salivary effects on microbial binding and their accumulation to the surface as well as inter-species interaction, there has not been considerable attention on the fitness and behavior of $S$. mutans and $C$. albicans during biofilm development in human saliva in the context of ECC.

Here, we sought to investigate how $S$. mutans and C. albicans form mixed-species biofilm and enhance the biofilm virulence in the presence of saliva. To understand the effect of saliva on biofilm behaviors, we comprehensively compared the properties of S. mutans single-species (Sm) and S. mutans-C. albicans crosskingdom (Sm-Ca) biofilms cultured in saliva-supplemented media $(0-100 \%)$. By determining the enamel dissolution caused by these biofilms using ultra-precision surface topography scanning microscopy, we attempted to understand the enhanced virulence of the cross-kingdom biofilms in the context of dental caries. In addition, by testing a hyphal deficient C. albicans mutant strain and clinical isolates, we sought to understand how hyphal transformation of C. albicans affects the biofilm's acidogenicity when cultured with $S$. mutans in the presence of saliva. The data reveal an important feature of cross-kingdom biofilm interaction in human saliva, explaining in part why and how these synergistic interactions are deeply involved in ECC.

\section{MATERIALS AND METHODS}

\section{Microorganisms and Culture Conditions}

Candida albicans SC5314, a well-characterized fungal strain, and Streptococcus mutans UA159, a proven virulent cariogenic dental pathogen and well-characterized EPS producer, were used for biofilm experiments. C. albicans SN152 and its hyphae deficient strain $(e f g 1 \Delta \Delta)$, and two clinical isolates (UR13 and UR18, gift from Jin Xiao, University of Rochester) were also used for evaluating the role of hyphae in biofilm formation (see Supplementary Table S1 for C. albicans strains used in this study). Spider agar plates (1\% Difco nutrient broth, $1 \%$ mannitol, $0.2 \%$ dibasic potassium phosphate, $1.5 \%$ agar, $\mathrm{pH} 7.2$ ) were prepared and used to confirm hyphal deficiency of mutant and clinical isolates of C. albicans (Liu et al., 1994). Microbial stocks were stored at $-80^{\circ} \mathrm{C}$ in tryptic soy broth containing $50 \%$ glycerol before use. All strains were grown to mid-exponential phase (optical densities at $600 \mathrm{~nm}$ of 0.8 and 1.0 for C. albicans and $S$. mutans, respectively) in ultrafiltered $(10-\mathrm{kDa}$ molecular-mass cutoff; Millipore, Billerica, MA, USA) yeast-tryptone extract broth containing $2.5 \%$ tryptone and $1.5 \%$ yeast extract (UFYTE; pH 5.5 and 7.0 for C. albicans and S. mutans, respectively) with $1 \%$ (wt/ vol) glucose. Cells were harvested by centrifugation $(6,000 \mathrm{~g}$, $10 \mathrm{~min}, 4^{\circ} \mathrm{C}$ ) as described previously (Xiao et al., 2012).

\section{Saliva Collection}

The study protocol was reviewed and approved by the Institutional Review Board of the University of Pennsylvania (protocol \#818549). Written informed consent was obtained from all volunteers in this study. Saliva was collected from 4 different healthy individuals ( 3 females and 1 male) who did not have any medications recently for at least a month. Individual saliva donor chewed unflavored paraffin wax and saliva was collected in a conical tube on ice. This was performed in the morning without having breakfast. Collected saliva was centrifuged $\left(5,500 \mathrm{~g}, 4^{\circ} \mathrm{C}, 10 \mathrm{~min}\right)$, followed by filter sterilization $(0.22 \mu \mathrm{m}$ polyethersulfone (PES); ultra-low binding protein filter; Millipore, Billerica, MA). Filtered saliva was then kept in a $4^{\circ} \mathrm{C}$ refrigerator until use.

\section{In Vitro Biofilm Model}

Biofilms were formed using our saliva-coated hydroxyapatite (sHA) model as described previously (Koo et al., 2010; Xiao et al., 2012; Falsetta et al., 2014). HA discs (diameter, $1.25 \mathrm{~cm}$; surface area, $2.7 \pm 0.2 \mathrm{~cm}^{2}$; Clarkson Chromatography Products, Inc., South Williamsport, PA) were coated with filter-sterilized 
clarified human saliva using $0.22 \mu \mathrm{m}$ PES filter (Millipore, Billerica, MA) as described previously (Xiao et al., 2012). The HA discs were vertically suspended in 24-well plates using a custom-made wire disc holder, mimicking the free smooth surfaces of the pelliclecoated teeth (Koo et al., 2010; Xiao et al., 2012).

To prepare biofilm culture media in which the proportion of saliva increased in a dose-dependent manner, UFYTE culture medium and filter sterilized saliva were mixed in various ratios; UFYTE:saliva (v/v) 100:0 $\left(\mathrm{SAL}_{0}\right), 25: 75\left(\mathrm{SAL}_{25}\right)$, 50:50 $\left(\mathrm{SAL}_{50}\right)$, and $0: 100\left(\mathrm{SAL}_{100}\right)$. Each disc was inoculated with i) approximately $2 \times 10^{6} \mathrm{CFU}$ of $S$. mutans/ml or ii) $\sim 2 \times 10^{4}$ $\mathrm{CFU}$ of C. albicans/ml [containing predominantly yeast cell forms (Gregoire et al., 2011)] or iii) both $\sim 2 \times 10^{6}$ CFU of $S$. mutans $/ \mathrm{ml}$ and $\sim 2 \times 10^{4} \mathrm{CFU}$ of $C$. albicans $/ \mathrm{ml}$ in a prepared culture medium $\left(\mathrm{SAL}_{0}\right.$ to $\left.\mathrm{SAL}_{100}\right)$ supplemented with $1 \%(\mathrm{w} / \mathrm{v})$ sucrose at $37^{\circ} \mathrm{C}$ under $5 \% \mathrm{CO}_{2}$; the proportion of the microorganisms in the inoculum is similar to that found in plaque samples from children with ECC (de Carvalho et al., 2006). The culture medium was changed twice daily at 8 am and $6 \mathrm{pm}$ and $\mathrm{pH}$ of the supernatant was determined using an Orion $\mathrm{pH}$ electrode attached to an Orion DUAL STAR $^{\mathrm{TM}} \mathrm{pH}$ meter (Thermo Fischer Scientific) until the end of the experimental period $(42 \mathrm{~h})$. The biofilms were collected at $18 \mathrm{~h}$ and $42 \mathrm{~h}$ for imaging and biochemical analysis. At least 3 independent biofilm experiments were performed for each of the conditions.

\section{Microbiological and Biochemical Biofilm Analysis}

Collected biofilms at each time point were subjected to standard microbiological and biochemical analysis. Briefly, the biofilms were removed and homogenized by sonication, and the number of viable cells (total number of CFU per biofilm) was determined (Cocco et al., 2020). In parallel, an aliquot of biofilm suspension was centrifuged $\left(5,500 \mathrm{~g}, 10 \mathrm{~min}, 4^{\circ} \mathrm{C}\right)$, and the pellet was washed twice with Milli-Q water, dried in an oven $\left(105^{\circ} \mathrm{C}, 24 \mathrm{~h}\right)$, and weighed. Quantification of polysaccharides was performed using an established colorimetric (phenol-sulfuric acid method) assay detailed previously (Klein et al., 2012; Xiao et al., 2012; Falsetta et al., 2014). At least 3 independent biofilm experiments were performed for each of the assays.

To monitor extracellular $\mathrm{pH}$ gradients in real-time, $100 \mu \mathrm{l}$ of the supernatant was collected from biofilm culture media and measured $\mathrm{pH}$ at every hour during the middle phase of biofilm culture $(18-28 \mathrm{~h})$. In order to harvest the supernatant without affecting biofilm growth nor mechanically disturbing the biofilm, 6 replicates were used. The supernatants were collected from two biofilm samples sequentially at every hour. Total volume of the supernatant collected per biofilm sample does not exceed $300 \mu \mathrm{l}$, so that vertically standing sHA discs were not exposed out of the interface of the culture medium.

\section{Confocal Microscopy Analysis}

The biofilms formed in each condition were examined using confocal laser scanning microscopy (CLSM) combined with quantitative computational analysis. Briefly, S. mutans cells were stained with $2.5 \mu \mathrm{M}$ SYTO 9 green-fluorescent nucleic acid stain (485/498 nm; Molecular Probes Inc., Eugene, OR, USA) and C. albicans cells were stained with concanavalin A (ConA) lectin conjugated with tetramethylrhodamine at $40 \mu \mathrm{g} /$ $\mathrm{ml}$ (555/580 nm; Molecular Probes, Inc.), while EPS glucans were labeled with $1 \mu \mathrm{M}$ Alexa Fluor 647-dextran conjugate (647/668 $\mathrm{nm}$; Molecular Probes Inc.) as detailed previously (Xiao et al., 2012; Falsetta et al., 2014). The confocal images of biofilms were obtained using an upright single-photon confocal microscope (LSM800, Zeiss, Jena, Germany) with a $20 \times$ (numerical aperture, 1.0) water objective. Each component was illuminated sequentially to minimize cross-talk as follow: SYTO 9 ( $S$. mutans) was excited using $488 \mathrm{~nm}$ and was collected by a $480 /$ $40 \mathrm{~nm}$ emission filter; ConA (C. albicans) was excited using 560 $\mathrm{nm}$, and was collected by a 560/40 nm emission filter; Alexa Fluor 647 (EPS) was excited using $640 \mathrm{~nm}$ and collected by a $670 /$ $40 \mathrm{~nm}$ emission filter.

To track microbial colonization and subsequent biofilm formation cultured in saliva, biofilm images were taken at 2, 4, 6,8 , and $18 \mathrm{~h}$ after seeding microorganisms on the sHA discs in $\mathrm{SAL}_{100}$ media. Taken images at each time point were subjected to the quantification of biofilm biomass and visualization. Briefly, image stacks for each channel obtained using a Zeiss LSM800 were converted to 8-bit ome.tiff files and the COMSTAT2 plugin of ImageJ was used to generate values for biovolume $\left(\mu \mathrm{m}^{3} / \mu \mathrm{m}^{2}\right)$. Biovolumes of $S$. mutans, C. albicans, and EPS glucans were quantified using COMSTAT2 as detailed elsewhere (Heydorn et al., 2000; Vorregaard, 2008; Kim et al., 2018). Biovolume values for each channel and total biomass up to $8 \mathrm{~h}$ were fit to the power-law $-\mathrm{V}(\mathrm{t})=\mathrm{a} \times \mathrm{t}^{\mathrm{b}}$, where $\mathrm{a}$ and $\mathrm{b}$ are constants for $\mathrm{a}$ particular curve (Paula et al., 2020). At least 3 independent biofilm experiments were performed for each of the analysis.

\section{Enamel Surface Demineralization}

Sterilized human tooth enamel blocks were coated with sterile clarified saliva (sTE, $4 \mathrm{~mm} \times 4 \mathrm{~mm}$ ). $\sim 2 \times 10^{6} \mathrm{CFU} / \mathrm{ml}$ of $S$. mutans and $\sim 2 \times 10^{4} \mathrm{CFU} / \mathrm{ml}$ of $C$. albicans were grown on sTE in $\mathrm{SAL}_{100}$ containing $1 \%$ sucrose $(\mathrm{w} / \mathrm{v})$. Biofilms were formed on enamel blocks mounted vertically at $37^{\circ} \mathrm{C}$ in $5 \% \mathrm{CO}_{2}$ for $114 \mathrm{~h}$ as described elsewhere (Koo et al., 2010; Liu et al., 2018). SAL 100 was replaced twice daily. Biofilms were collected at $114 \mathrm{~h}$ for standard microbiological and biochemical analysis, and the enamel slabs were collected for topography and surface roughness measurement. The surface topography and roughness of the enamel surface were analyzed by a nondestructive confocal contrasting method using Zeiss LSM 800 with a C Epiplan-Apochromat 50× (numerical aperture, $0.95)$ nonimmersion objective. The images were processed using ConfoMap (Zeiss) to create $3 \mathrm{D}$ topography rendering and measure the surface properties in $3 \mathrm{D}$. To quantify the surface demineralization, 3D surface roughness parameters arithmetical mean height $\left(S_{\mathrm{a}}\right)$, maximum peak height $\left(S_{\mathrm{p}}\right)$, and maximum pit height $\left(S_{\mathrm{v}}\right)$ were measured using ISO 25178 (ISO, 2012). At least 3 independent experiments were performed for the assay.

\section{Statistical Analyses}

All statistical analyses for biochemical, microbiological, biovolume, and topographical data were carried out using 
GraphPad Prism 8 via i) analysis of variance (ANOVA), followed by Dunnett's test for post-hoc analysis or ii) Student's t-test where appropriate. The level of significance was set at $5 \%$.

\section{RESULTS}

\section{Effects of Human Saliva on the Microbiological and Biochemical Properties of Biofilms}

S. mutans (Sm) and C. albicans (Ca) exhibit a mutually symbiotic relationship to amplify the virulence of plaque-biofilms (Falsetta et al., 2014; Hwang et al., 2017). However, it remains largely unknown how these organisms modulate cross-kingdom biofilm formation and enhance its virulence in human saliva. To investigate the effect of human saliva on the formation and development of Sm and Sm-Ca biofilms, we prepared bacterial culture media in which the proportion of saliva increased in a dose-dependent manner $\left(\mathrm{SAL}_{0}\right.$ to $\left.\mathrm{SAL}_{100}\right)$ and performed biofilm analysis (Figure 1A). Clarified human saliva was collected from four donors which presented similar $\mathrm{pH}$ buffering capacities (Supplementary Figure S1). Dry-weight, EPS, and CFU were assessed individually for each donor saliva ( $\mathrm{n} \geq 3$; Supplementary Figures S2-S5), and then the average values were calculated (Figures 1B-D). We observed that biofilm properties ( $\mathrm{pH}$ of biofilm supernatant, dry-weight, and CFU) tested by individual saliva were also similar (Supplementary
Figures S2-S5). As shown in Figure 1, the dry-weight of Sm biofilm decreased in a dose-dependent manner as saliva content increased and only a negligible amount of biofilm biomass was detected when cultured in $100 \%$ clarified human saliva $\left(\mathrm{SAL}_{100}\right)$. However, in the Sm-Ca biofilm, the dry-weight remained unchanged up to $50 \%$ of saliva content. Although the dry-weight of Sm-Ca biofilm was reduced significantly in $\mathrm{SAL}_{100}$ (vs. 100\% UFYTE medium; $\mathrm{SAL}_{0}$ ), a considerable amount of biomass was detected (Figure 1B). Total insoluble EPS-glucan (Figure 1C) and CFU (Figure 1D) also showed similar trends. Note that the biomass of Sm biofilm in $\mathrm{SAL}_{100}$ was below the detection limit, thus it was not able to measure the content of EPS glucans in Sm cultured in $\mathrm{SAL}_{100}$. Additionally, we investigated the properties of $C$. albicans single-species biofilms in $\mathrm{SAL}_{0}-\mathrm{SAL}_{100}$. The data showed that $C$. albicans alone did not form robust biofilm nor induce acidic $\mathrm{pH}$ under all experimental conditions (regardless of saliva concentration in media) (Supplementary Figure S6).

\section{Effect of Saliva on the pH of Biofilm Supernatant}

Dropping of salivary $\mathrm{pH}$ under 5.5 is critical for tooth demineralization (Larsen and Pearce, 1997). Thus, we investigated the $\mathrm{pHs}$ of the biofilm supernatant throughout the biofilm experimental period (at 18, 28, $42 \mathrm{~h}$ ). Sm and Sm-Ca biofilms showed very similar levels of $\mathrm{pHs}$ in the $\mathrm{SAL}_{0}$ media and saliva partially added culture media $\left(\mathrm{SAL}_{25}\right.$ and $\left.\mathrm{SAL}_{50}\right)$. However,

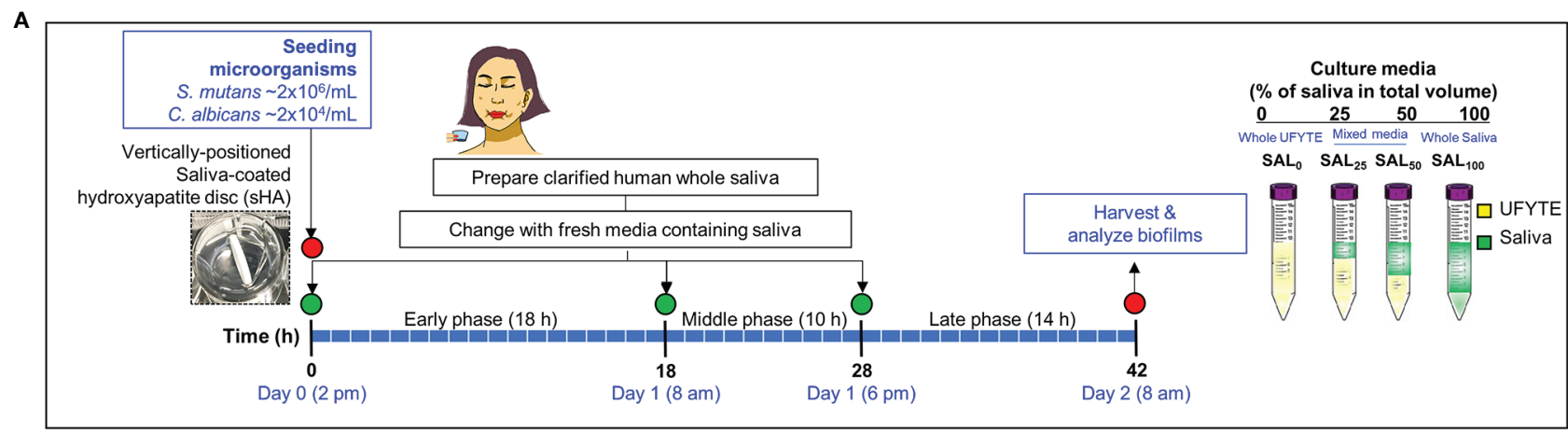

B

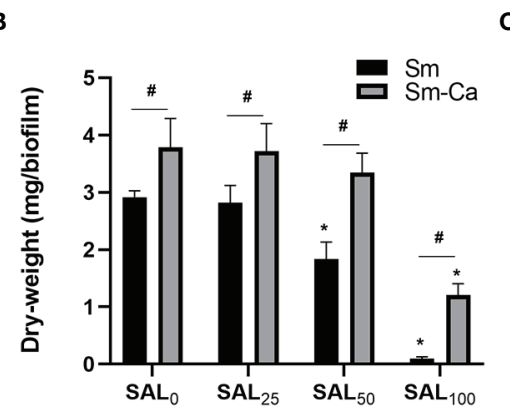

C

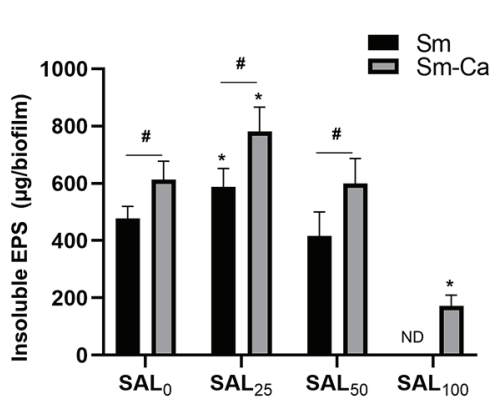

D

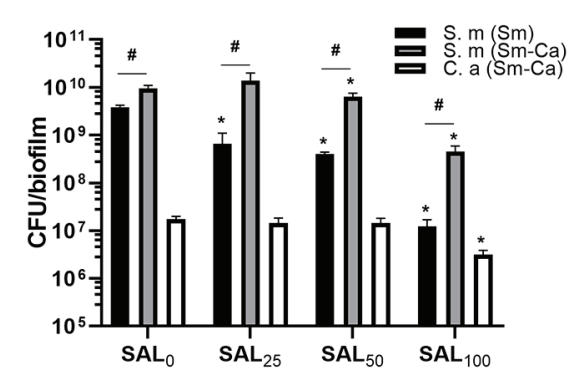

FIGURE 1 | Microbiological and biochemical properties of Sm and Sm-Ca biofilms in saliva contained media (0-100\%). (A) Biofilm experimental design and composition of culture media. (B) Biomass (Dry-weight) of biofilms. (C) Amounts of insoluble polysaccharides (EPS) in biofilms. The biomass of Sm biofilm in SAL 100 was below the detection limit, thus it was not able to measure the content of EPS-glucans in Sm cultured in SAL 100 . (D) CFU of $S$. mutans and C. albicans in Sm and Sm-Ca biofilms at final phases (42 h). Dry weight, EPS, and CFU experiments were performed ( $\mathrm{n}>3$ ) separately for each donor saliva, and then the average values were calculated. Asterisk indicates that the $p$-values are significantly different from $S A L_{0}$ (whole UFYTE) (ANOVA with Dunnett; $\left.{ }^{*} P<0.05\right)$. Hash indicates that the $p$-values are significantly different between two groups (Student's $t$-test; ${ }^{\#} P<0.05$ ). ND indicates not detected. 
the pHs of biofilm supernatant in $\mathrm{SAL}_{100}$ showed a completely different pattern between Sm and Sm-Ca biofilms (Figures 2A, B). Sm-Ca biofilm dropped the $\mathrm{pH}$ of biofilm supernatant ( 4.3), which was consistent with other cultivation conditions (i.e., $\mathrm{SAL}_{0}-\mathrm{SAL}_{50}$ ), while $\mathrm{Sm}$ biofilm maintained substantially higher $\mathrm{pH}$ than the critical point $(\mathrm{pH} 5.5)$ that allows enamel demineralization at all time points throughout the biofilm culturing period. Besides, we tracked the dynamic changes of the $\mathrm{pH}$ of biofilm supernatant to better understand how these Sm and Sm-Ca biofilms behave in $\mathrm{SAL}_{0}$ or $\mathrm{SAL}_{100}$ toward biofilm maturation (18-28 h; between 8 am and $6 \mathrm{pm}$ on the Day 2). In $\mathrm{SAL}_{0}, \mathrm{Sm}$-Ca biofilm more rapidly lowered the $\mathrm{pH}$ of biofilm supernatant (vs. Sm), while the $\mathrm{pH}$ of both biofilm supernatants reached to similar $\mathrm{pH}(\sim 4.3)$ at $28 \mathrm{~h}$ (Figure 2C). In $\mathrm{SAL}_{100}$, Sm-Ca biofilm slowly lowered the $\mathrm{pH}$ (vs. $\mathrm{SAL}_{0}$ ) but ultimately reached below $\mathrm{pH} 5$ at $26 \mathrm{~h}$. Although the $\mathrm{pH}$ of the Sm biofilm supernatant steadily decreased, it mainly remained in neutral $\mathrm{pH}(\sim 7)$ even at $28 \mathrm{~h}$ (Figure 2D).

\section{Structural Analysis of $\mathrm{Sm}$ and $\mathrm{Sm}-\mathrm{Ca}$ Biofilms}

As shown in Figures 1 and 2, we observed that only Sm-Ca coculture resulted in significant biofilm biomass and acidified biofilm supernatant. To further understand differences in biofilm properties between $\mathrm{Sm}$ and $\mathrm{Sm}$-Ca biofilms, we investigated the microbial growth and tertiary structure of Sm and $\mathrm{Sm}-\mathrm{Ca}$ biofilms cultured in $\mathrm{SAL}_{100}$ using a confocal microscope (Figure 3A). We did not observe a significant difference in $S$. mutans initial binding to the sHA disc between $\mathrm{Sm}$ and Sm-Ca biofilms at $2 \mathrm{~h}$ (Figure 3A). However, we observed distinct growth kinetics of S. mutans in Sm and SmCa biofilms at the early stage of biofilm formation ( $4 \mathrm{~h}$ in Figure 3, $p$-value: 0.005 ). This distinct growth kinetics of $S$. mutans between $\mathrm{Sm}$ and $\mathrm{Sm}$-Ca biofilms were more pronounced over time (Figures 3A, B). Indeed, S. mutans biovolume in Sm-Ca biofilm $\left(2.11 \mu \mathrm{m}^{3} / \mu \mathrm{m}^{2}\right)$ was approximately 2.5 -fold higher than that of Sm biofilm $\left(0.91 \mu \mathrm{m}^{3} / \mu \mathrm{m}^{2}\right)$ at $4 \mathrm{~h}$ (Figure 3B). We also observed the explosive production of EPS from Sm-Ca biofilms (Figure 3C). After $6 \mathrm{~h}$ of inoculation, Sm biofilm still showed only a few numbers of microcolonies with negligible EPS on the sHA disc, while a large number of $S$. mutans microcolonies enmeshed with EPS formed on the sHA disc were observed from Sm-Ca (Figure 3A). Growth of C. albicans also showed a similar trend with $S$. mutans growth and EPS production (Figures 3A-D), resulting in $\sim 12$-fold higher total biomass of Sm-Ca at $18 \mathrm{~h}$ (vs. Sm).

We have previously shown that biofilm growth dynamics can be fit to power-law curves as a form of $\mathrm{V}(\mathrm{t})=\mathrm{a} \times \mathrm{t}^{\mathrm{b}}$ (Paula et al., 2020), where $V(t)$ is the biovolume at time $t$, and $a$ and $b$ are
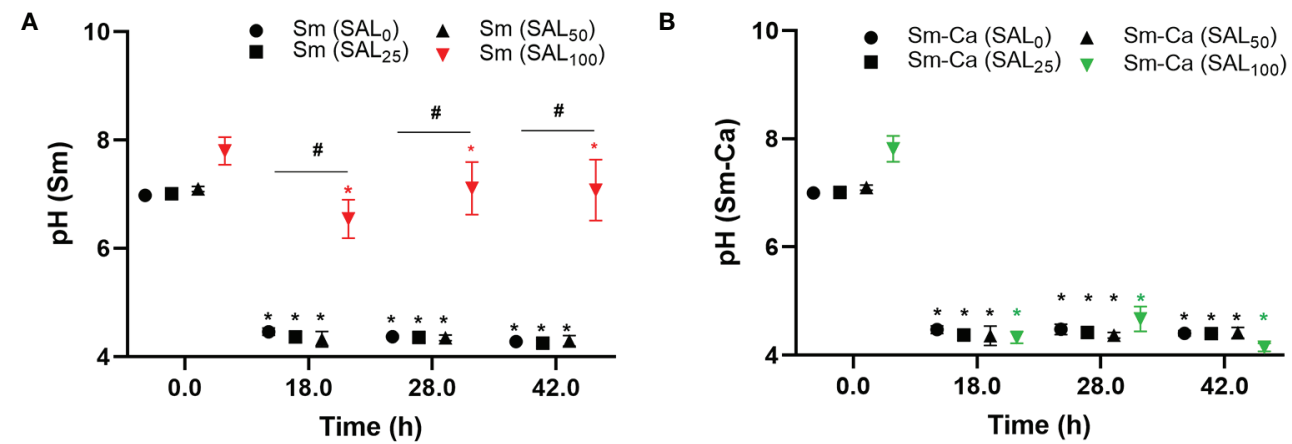

C

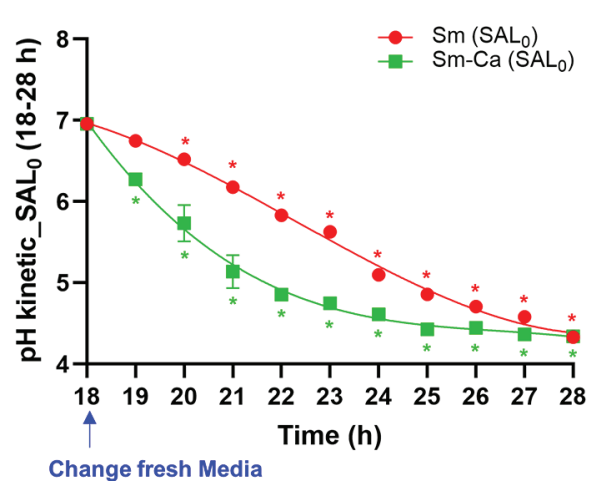

D

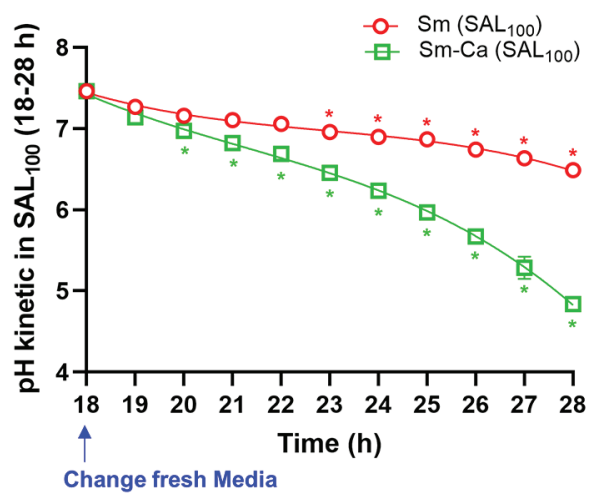

FIGURE 2 | Effect of human saliva on the pH of biofim supernatants in Sm and Sm-Ca biofilms. pH value at early (18 h), middle (28 h), and late (42 h) phases of Sm (A) and Sm-Ca (B) biofilms in different saliva ratios. Sm and Sm-Ca showed similar pH patterns under most culture conditions ( $\left(\mathrm{SAL}_{0}, \mathrm{SAL}_{25}\right.$, and $\left.\mathrm{SAL}_{50}\right)$, while $\mathrm{Sm}$ biofilm showed a completely different $\mathrm{pH}$ pattern when cultured under $\mathrm{SAL}_{100}$. Time-lapsed pH measurements of Sm and Sm-Ca biofilms in SAL (C) and SAL (D) during the middle phase (from $18 \mathrm{~h}$ to $28 \mathrm{~h}$ ). Asterisk indicates that the $p$-values are significantly different from the pH of the starting point (ANOVA with Dunnett; $\left.{ }^{*} P<0.05\right)$. Hash indicates that the $p$-values are significantly different among groups (Student's $t$-test; ${ }^{\#} P<0.05$ ). 

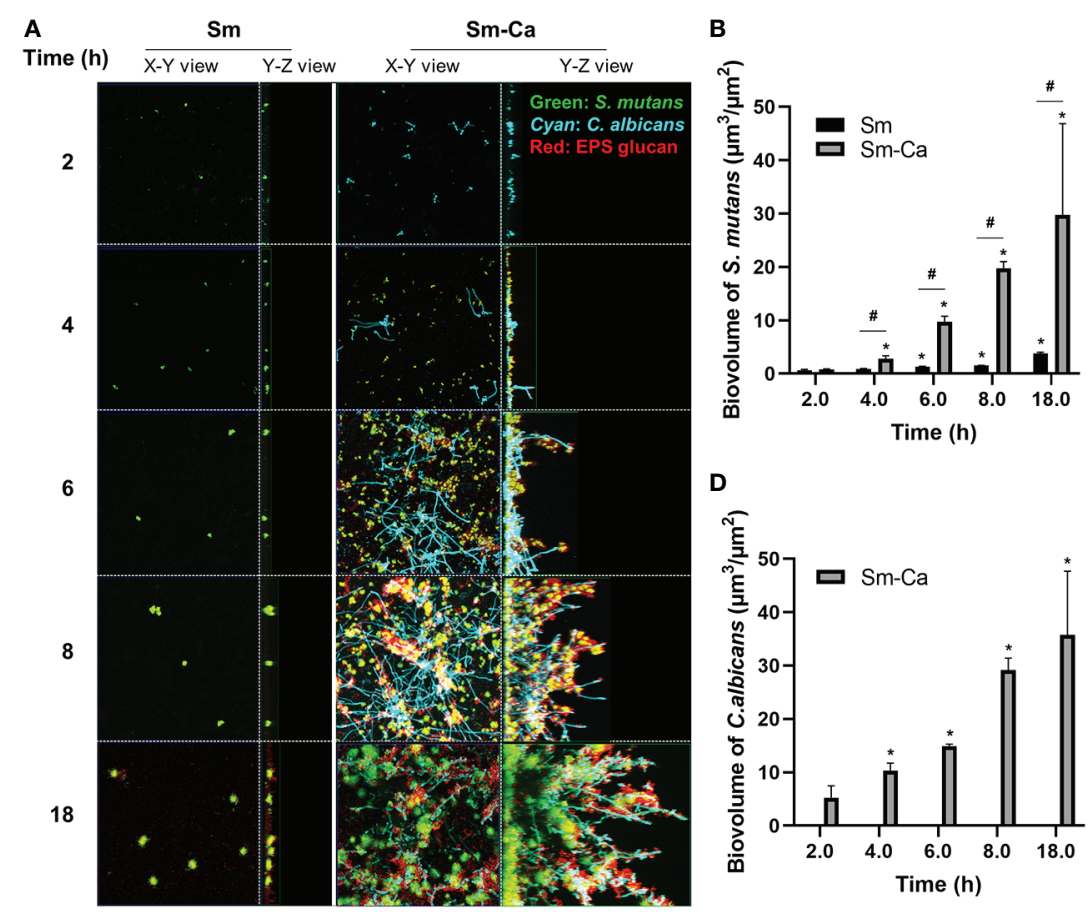

C
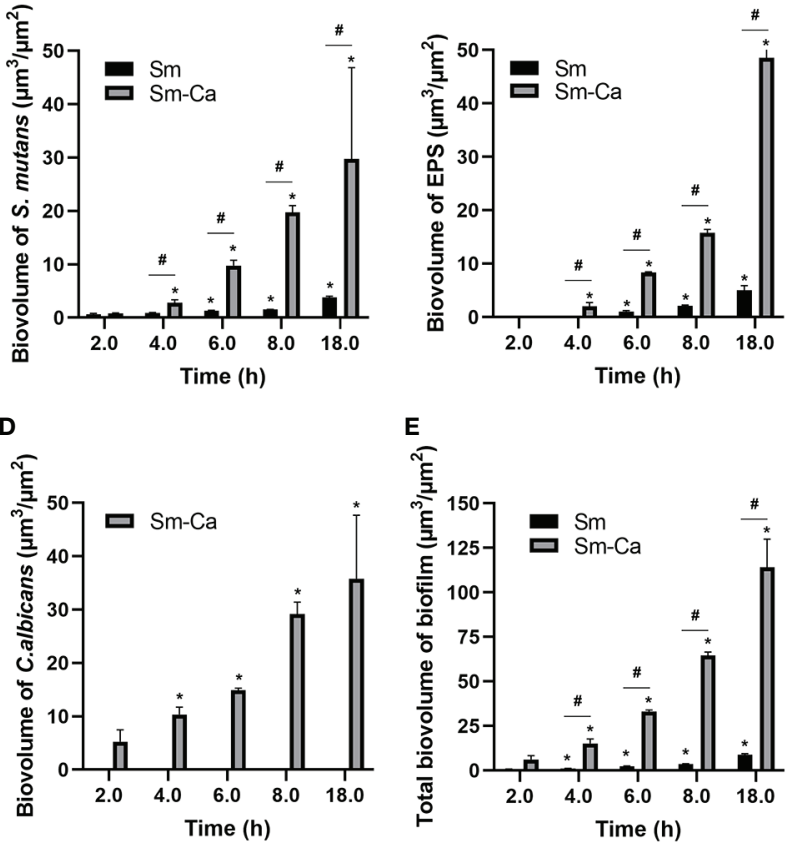

FIGURE 3 | Confocal images of biofilms and quantification of biofilm components. (A) Representative top (X-Y) and orthogonal (Y-Z) views of confocal images of Sm and Sm-Ca biofilms at 2, 4, 6, 8, and $18 \mathrm{~h}$ cultured under SAL $\mathrm{S}_{100}$. Bacterial cells are labeled with SYTO 9 (green), fungal cells with concanavalin Atetramethylrhodamine (Cyan), and EPS $\alpha$-glucan with Alexa Fluor 647 (red). Quantified biovolume of (B) S. mutans, (C) EPS, (D) C. albicans, and (E) total (sum of S. mutans, $C$. albicans, and EPS) in the biofilm. Asterisk indicates that the $p$-values are significantly different from the biovolume of $2 \mathrm{~h}$ (ANOVA with Dunnett; ${ }^{*}<<$ 0.05). Hash indicates that the $p$-values are significantly different between two groups $\left(t\right.$-test $\left.{ }^{\#} P<0.05\right)$.

normalization and exponent constants of the curve, respectively. To further elucidate the synergistic interactions in $\mathrm{Sm}-\mathrm{Ca}$ biofilms, we fitted the biovolume data to the power-law. Table 1 depicted values for the rate of change of $V(t)$ to highlight the rate of growth, and constants $\mathrm{a}$ and $\mathrm{b}$ of the curves (up to $8 \mathrm{~h}$ ). As shown, it is clear that the exponent constant, b, for Sm was 3.5 times higher in Sm-Ca biofilms than in Sm biofilms. This constant for Sm in single biofilms was under 1 , indicating retardation of $S$. mutans growth in $\mathrm{SAL}_{100}$. Lastly, the overall constants, $a$ and $b$, for total biomass in Sm-Ca biofilms were $\sim 7$ and $\sim 1.3$ times higher, respectively (vs. a and b for total biomass in Sm biofilms).

Next, we observed the morphology of $\mathrm{Sm}$ and $\mathrm{Sm}-\mathrm{Ca}$ biofilms. Interestingly, the hyphal transition of surface-bound C. albicans was initiated from $2 \mathrm{~h}$ and these hyphal cells were elongated over time (Figure 3A). Particularly, S. mutans microcolonies were harmonized with these C. albicans in SmCa biofilm, showing numerous small $S$. mutans microcolonies formed along the hyphae (Figure 3A, Supplementary Figure S7). The data revealed that $\mathrm{Sm}-\mathrm{Ca}$ synergistic interaction

TABLE 1 | Quantified biofilm components of S. mutans single-species and S. mutans-C. albicans mixed-species biofilms and corresponding power-law parameters.

\begin{tabular}{|c|c|c|c|c|c|c|c|c|c|c|c|c|c|}
\hline \multirow[b]{2}{*}{ Sm } & \multirow[b]{2}{*}{$\begin{array}{c}\text { Time }(h) \\
2\end{array}$} & \multicolumn{3}{|c|}{ S. mutans } & \multicolumn{3}{|c|}{ EPS } & \multicolumn{3}{|c|}{ C. albicans } & \multicolumn{3}{|c|}{ Total biomass } \\
\hline & & $\begin{array}{c}\Delta \mathrm{V}(\mathrm{t}) / \Delta \mathrm{t} \\
0.31\end{array}$ & $a$ & $b$ & $\begin{array}{c}\Delta \mathrm{V}(\mathrm{t}) / \Delta \mathrm{t} \\
0.01\end{array}$ & a & $b$ & $\Delta \mathrm{V}(\mathrm{t}) / \Delta \mathrm{t}$ & a & $\mathrm{b}$ & $\begin{array}{c}\Delta \mathrm{V}(\mathrm{t}) / \Delta \mathrm{t} \\
0.32\end{array}$ & a & $b$ \\
\hline & 4 & 0.14 & 0.39 & 0.65 & 0.05 & $6.0 \mathrm{E}-4$ & 3.94 & & & & 0.19 & 0.23 & 1.27 \\
\hline & 6 & 0.20 & & & 0.48 & & & & & & 0.68 & & \\
\hline & 8 & 0.09 & & & 0.53 & & & & & & 0.62 & & \\
\hline & 18 & 0.22 & & & 0.29 & & & & & & 0.52 & & \\
\hline $\mathrm{Sm}-\mathrm{Ca}$ & $\begin{array}{c}\text { Time }(h) \\
2\end{array}$ & $\begin{array}{c}\Delta \mathrm{V}(\mathrm{t}) / \Delta \mathrm{t} \\
0.32\end{array}$ & $a$ & $b$ & $\begin{array}{c}\Delta \mathrm{V}(\mathrm{t}) / \Delta \mathrm{t} \\
0.03\end{array}$ & a & $b$ & $\begin{array}{c}\Delta \mathrm{V}(\mathrm{t}) / \Delta \mathrm{t} \\
2.64\end{array}$ & a & $\mathrm{b}$ & $\begin{array}{c}\Delta \mathrm{V}(\mathrm{t}) / \Delta \mathrm{t} \\
2.99\end{array}$ & a & $b$ \\
\hline & 4 & 0.74 & 0.11 & 2.29 & 0.98 & 3.8E-3 & 4.19 & 2.53 & 2.21 & 1.16 & 4.25 & 1.75 & 1.63 \\
\hline & 6 & 2.38 & & & 3.18 & & & 2.27 & & & 7.83 & & \\
\hline & 8 & 4.32 & & & 3.68 & & & 7.13 & & & 15.13 & & \\
\hline & 18 & 1.00 & & & 3.28 & & & 0.67 & & & 4.94 & & \\
\hline
\end{tabular}


promoted initial biofilm structuring and subsequent development in human saliva.

\section{Effect of Hyphal Formation Capability of $\mathbf{C}$. albicans on Acidogenicity of Cross- kingdom Biofilm}

Facilitated hyphal elongation of C. albicans and the formation of S. mutans microcolonies along the hyphae in $\mathrm{SAL}_{100}$ may enhance the acidogenicity of Sm-Ca biofilm (Figure 2). To investigate the role of the hyphal formation capability of $C$. albicans on Sm-Ca biofilm development in $\mathrm{SAL}_{100}$, we compared the biofilm properties between $C$. albicans wild type (Ca_WT; SC5314 or SN152) and its hyphal-deficient mutant strain EFG1 (Ca_efg1 $\Delta \Delta$ ) (Supplementary Table S1). First, we confirmed the hyphal formation ability Ca_efg1 $1 \Delta$ using spider agar plates. As expected, Ca_WT formed dense hyphae, while the Ca_efg1 $\Delta \Delta$ strain did not form hyphae at all on spider agar plates (Figure 4A). We also examined the morphologies of biofilms formed by these two C. albicans strains and S. mutans. Representative confocal images of $18 \mathrm{~h}$ biofilm clearly showed that Ca_efg1 $\Delta \Delta$ strain has no ability to transform yeast to hyphae even when cultured with $S$. mutans in $\mathrm{SAL}_{100}$. In contrast, Sm-Ca_WT showed abundant long-hyphae across the surface (Figure 4B). Also, Sm-Ca_efg1 $\Delta \Delta$ formed a flat and thinner biofilm in $\mathrm{SAL}_{100}$, resulting in significantly less biomass (vs. Sm-Ca_WT; Figure 4D). Nevertheless, there were no statistical differences in CFU and more importantly $\mathrm{pH}$ profiles, regardless of strains (Figure 4C).

To gain further insight into the effect of hyphal transition on the biofilm formation and acidogenicity, we also tested two clinical isolates of C. albicans collected from ECC patients. We selected these two isolates as it showed clear differences in hyphal development efficiencies (UR13: long hyphae; UR18: short hyphae on spider agar plates, Figure 4A). Both Sm-Ca_UR13 and Sm-Ca_UR18 exhibited acidic microenvironment $(\mathrm{pH}<4.5)$, similar to other cross-kingdom biofilm combinations (Figure 4C). Dry-weight of biofilms formed with clinical isolates also showed a similar level to the one from Sm-Ca_WT (Figure 4D). Interestingly, S. mutans population in Sm-Ca_UR13 (or SmCa_UR18) was approximately a log higher than other combinations, while there were no significant differences in $C$. albicans populations (Figure $4 \mathbf{E}$ ). The data indicated that the lack of yeast-to-hyphae transition of C. albicans in the crosskingdom biofilm did not compromise the acidogenicity of biofilms.

\section{Demineralization of Human Enamel Surface by Biofilms Cultured in Human Saliva}

Finally, we compared the level of enamel demineralization by culturing each biofilm on the human enamel slab in $\mathrm{SAL}_{100}$ supplemented with $1 \%$ sucrose. As shown in Figure $\mathbf{5 A}$, we
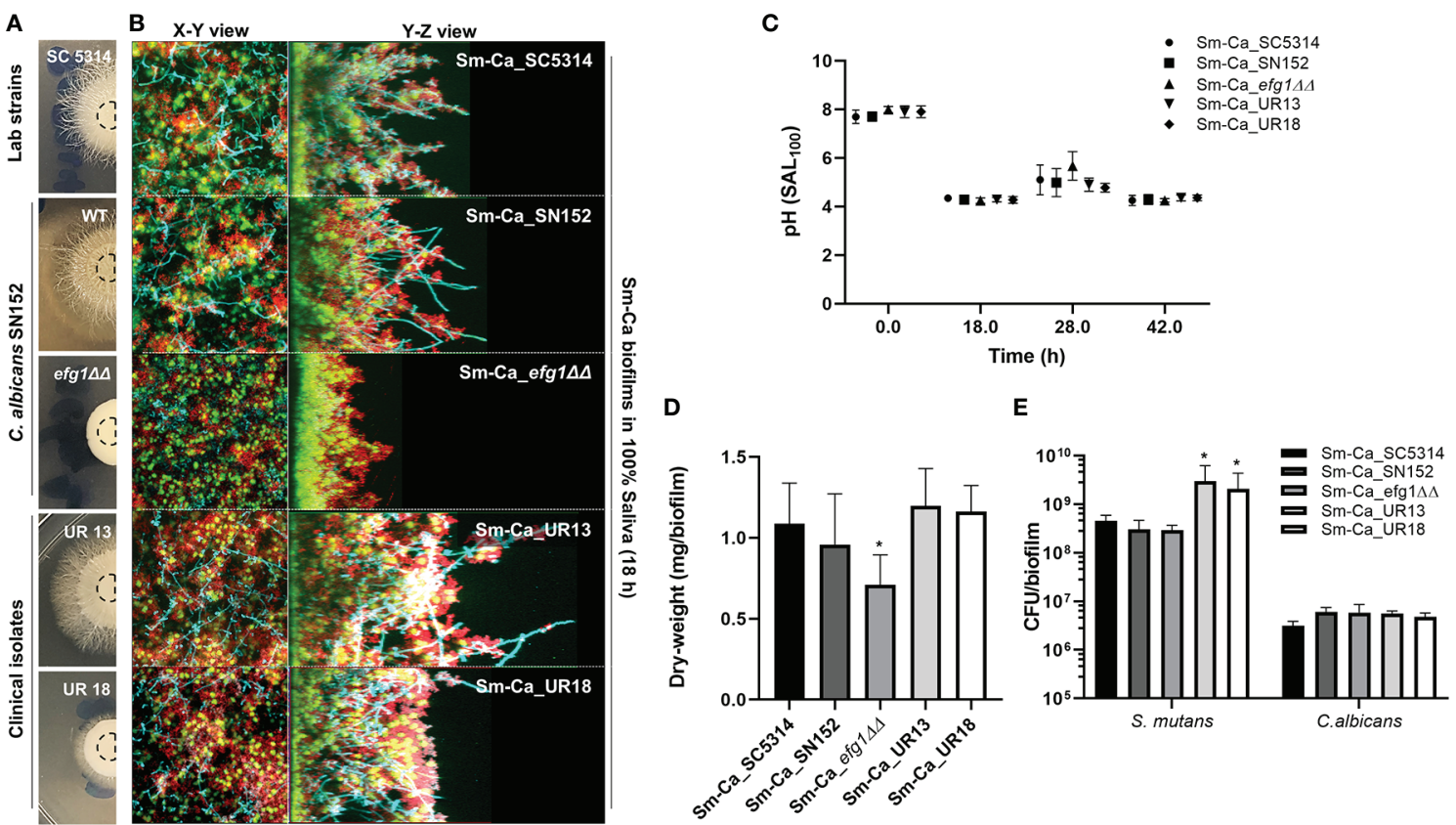

FIGURE 4 | Effect of hyphal transformation ability on the properties of Sm-Ca biofilms in saliva. (A) C. albicans colonies of SC5314, SN152, efg144, UR13, and UR18 strains on Spider agar plate. Morphology of fungal colony was photographed after incubation for 4 days at $37^{\circ} \mathrm{C}$. (B) Representative top (X-Y) and orthogonal (Y-Z) views of confocal images of each mixed-species biofilm under SAL $\mathrm{L}_{100}$ at $18 \mathrm{~h}$. Bacterial cells are labeled with SYTO 9 (green), fungal cells with concanavalin Atetramethylrhodamine (Cyan), and EPS $\alpha$-glucan with Alexa Fluor 647 (red). (C) pH value at each time point (18, 28, and 42 h). Data were analyzed by descriptive analysis and one-way analysis of variance (ANOVA) using GraphPad Prism 8. (D) Total biomass (dry weight) of each Sm-Ca biofilm and (E) CFU of S. mutans and C. albicans at the endpoint (42 h). Asterisk indicates that the $p$-values are significantly different from wild type biofilm (Sm-Ca_SC5314) (ANOVA with Dunnett; $\left.{ }^{\star} P<0.05\right)$. 
observed a distinct difference in the $\mathrm{pH}$ of biofilm supernatant between Sm and Sm-Ca biofilms (Figure 5A). Sm biofilm hardly lowered the $\mathrm{pH}$ of biofilm supernatant as we observed from the previous experiment using HA disc. In contrast, $\mathrm{Sm}-\mathrm{Ca}$ biofilm gradually lowered the $\mathrm{pH}$ of biofilm supernatant over time; eventually, it reached acidic $\mathrm{pH}$ below 5.5 from $66 \mathrm{~h}$ till the end of the experiment (Figure 5D). By culturing biofilms for five days on human enamel slabs, we were able to have similar biofilm biomass and CFU to the one we observed from the HA disc model (Figures 4D, E and 5B, C). Such significant differences in $\mathrm{pH}$ and biofilm density between $\mathrm{Sm}$ and $\mathrm{Sm}-\mathrm{Ca}$ biofilms could inflict differential damage to the mineralized tooth tissue underneath the distinctive biofilms. Thus, we inspected the enamel destruction by biofilms visually and quantitatively using confocal surface topographical analysis. As shown in Figure 5D, the intact surface before forming biofilms showed a smooth and flat surface with subnanometer-level surface roughness (Figures 5D, E). We also observed mostly intact but mildly damaged surfaces where Sm biofilm was formed (Figures 5D, E). In marked contrast, the enamel surfaces accommodated Sm-Ca biofilm showed significantly eroded surfaces with microcavities

(Figure 5D). This observation was supported by quantitative analysis of arithmetical mean height $\left(S_{\mathrm{a}}\right)$, maximum peak height $\left(S_{\mathrm{p}}\right)$, and maximum pit height $\left(S_{\mathrm{v}}\right)$ following ISO 25178 (ISO, 2012). Overall, enamel surfaces degraded by Sm-Ca biofilms exhibited $\sim 6$-fold higher $S_{\mathrm{a}}, \sim 2.8$-fold higher $S_{\mathrm{p}}$, and $\sim 1.7$-fold higher $S_{\mathrm{v}}$, than those degraded by Sm biofilms (Table 2). Collectively, our findings clearly show that the symbiotic and synergistic interactions between $S$. mutans and C. albicans are critical to forming mature biofilms in human saliva that can result in localized enamel demineralization and tooth decay.

\section{DISCUSSION}

Plenty of studies have shown that plaque biofilms cause various infectious oral diseases in the human mouth (Marsh, 2010; Murakami et al., 2018). Specific to dental caries, high levels of

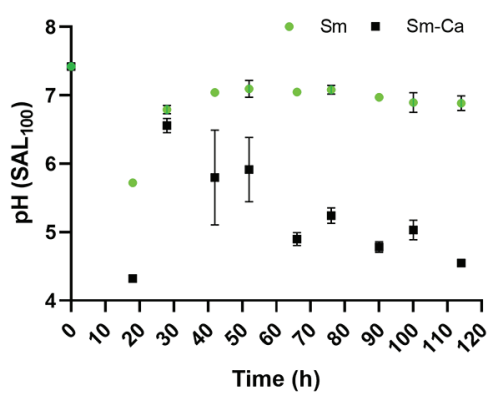

B

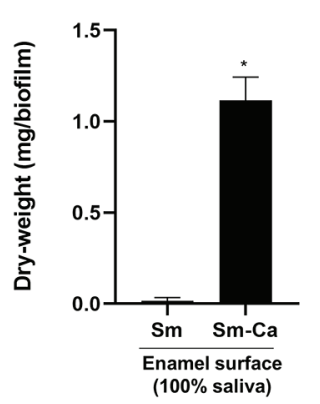

C

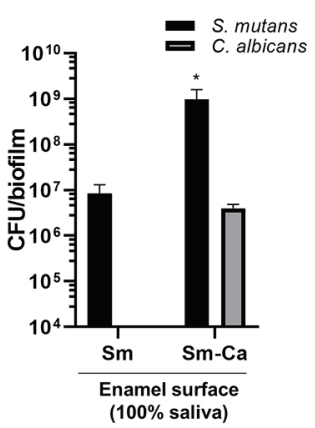

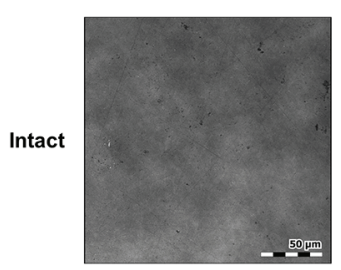
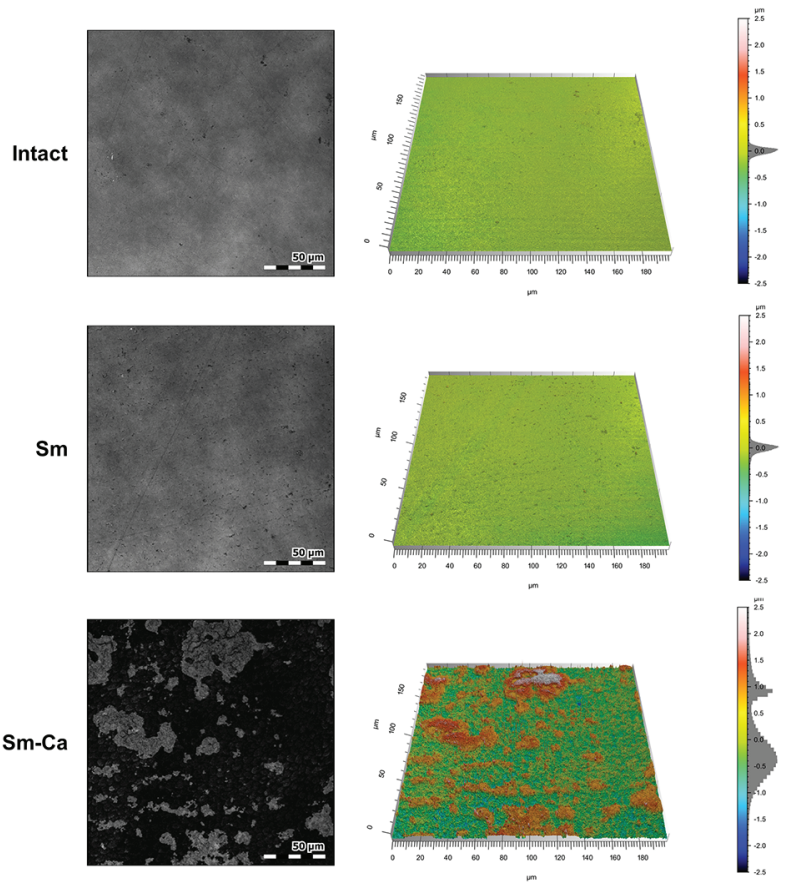

FIGURE 5 | Demineralization of human enamel surface by Sm and Sm-Ca biofilm in $\mathrm{SAL}_{100}$. (A) pH changes in Sm and Sm-Ca biofilm throughout the biofilm experiment. (B) Biomass (Dry-weight) of biofilms and (C) CFU of S. mutans and C. albicans in Sm and Sm-Ca biofilms at final phases (114 h). (D) Representative confocal surface-topography and roughness of enamel surfaces (Scale bar: $50 \mu \mathrm{m}$ ). Asterisk indicates that the $p$-values are significantly different from Sm biofilm (Student's $t$-test; $\left.{ }^{*} P<0.05\right)$.

TABLE 2 | Surface roughness parameters of the demineralization of human enamel by biofilms.

\begin{tabular}{lcrr}
\hline Surface Roughness Parameter $(\boldsymbol{\mu m})$ & Intact & Sm & Sm-Ca \\
\hline$S_{a}$ (arithmetical mean height) & $0.0388 \pm 0.0098$ & $0.1347 \pm 0.0521$ & $0.7880 \pm 0.5087$ \\
$S_{p}$ (maximum peak height) & $1.0810 \pm 0.5863$ & $3.6960 \pm 4.5459$ & $10.2978 \pm 2.8721$ \\
$S_{v}$ (maximum pit height) & $1.6482 \pm 1.1531$ & $3.6224 \pm 3.1856$ & $6.0872 \pm 3.2119$
\end{tabular}


S. mutans and C. albicans are found from plaque-biofilms in ECC patients (de Carvalho et al., 2006; Xiao et al., 2018). These microbes develop symbiotic cross-kingdom interactions that amplify the toxicity of plaque-biofilms (Falsetta et al., 2014; Hwang et al., 2017). Although saliva plays critical roles in the oral cavity such as inhibiting microbial activity or promoting microbial adhesion (Ahn et al., 2008; Cheaib et al., 2015), studies on the effect of saliva on the behaviors and fitness of crosskingdom biofilm have received limited attention.

Here, we compared the biofilm properties cultured in saliva contained media (0 to 100\%) and investigated how human saliva modulates the acidogenicity and virulence of cariogenic crosskingdom biofilms to mimic physiological conditions in the human mouth. While both Sm and Sm-Ca biofilms prospered and created acidic microenvironments either in an optimized culture medium $\left(\mathrm{SAL}_{0}\right)$ or saliva-supplemented media $\left(\mathrm{SAL}_{25}\right.$ or $\mathrm{SAL}_{50}$ ), Sm biofilm was more susceptible to saliva contents; dryweight started to substantially reduce when saliva proportion was higher than $50 \%$ and it was barely detected in the $100 \%$ clarified saliva without exhibiting acidogenicity (Figure 2A). Although, we also observed a significant reduction of dry-weight of Sm-Ca biofilm in $\mathrm{SAL}_{100}, \mathrm{Sm}$-Ca biofilm matured sufficiently to induce an acidic environment (Figure 2B). The data revealed distinct biofilm fitness in response to saliva concentration, thus it is imperative to more closely mimic clinically relevant conditions when performing in vitro study. By tracking initial settlement and colonization of microbes on the sHA discs over time, we found that the colonized S. mutans in Sm-Ca biofilm on the sHA disc were more rapidly grown in $\mathrm{SAL}_{100}$ (vs. Sm; Figure 3A). In turn, acidogenicity of the $\mathrm{Sm}$-Ca biofilm was reinforced, thereby incurring severe enamel dissolution (Figure 5D).

Saliva contains a wide variety of antimicrobial peptides (AMPs) that act as host defense molecules (such as defensins and histatins) to combat microbial infestations and challenges (Helmerhorst et al., 1999; Dale and Fredericks, 2005; Jurczak et al., 2015). Therefore, we examined whether the $100 \%$ clarified saliva could inhibit the viability and growth of $S$. mutans, thereby blocking them from forming mature biofilm in $\mathrm{SAL}_{100}$. Interestingly, human saliva was no harm to $S$. mutans in the planktonic phase. Rather, it showed a similar S. mutans population either in single-species or mixed-species cultivation (Supplementary Figure S8). A similar observation has been reported that $S$. mutans was resistant to AMPs in saliva (Phattarataratip et al., 2011). Although saliva did not affect the viability of $S$. mutans, $S$. mutans alone failed to form mature biofilm in $\mathrm{SAL}_{100}$, while significant biomass was detected from Sm-Ca biofilm under the same conditions (Figure 1B). These results suggest that saliva may affect further biofilm development on the surface. Dramatic increases in $\mathrm{V}(\mathrm{t}) / \Delta \mathrm{t}(>>1)$ of all biofilm components in $\mathrm{Sm}-\mathrm{Ca}$ indicate their explosive growth at the initial developmental phase. In contrast, $\mathrm{V}(\mathrm{t}) / \Delta \mathrm{t}$ of all those components in Sm showed significantly less than 1, indicating that their growth was saturating over time (Table 1). The data revealed that the co-existence of $S$. mutans and C. albicans significantly enhanced the growth kinetics of Sm-Ca biofilm in $\mathrm{SAL}_{100}$ (vs. Sm).
C. albicans undergoes a reversible morphological transition from single yeast cells to pseudohyphae and hyphae filaments (Carlisle and Kadosh, 2013). Hyphae of C. albicans are essential elements for the structural integrity to be fully developed biofilms with multilayered architectures (Baillie and Douglas, 1999; Kadosh, 2013). By investigating the properties of diverse cross-kingdom biofilms using hyphal deficient C. albicans strains, we confirmed that all the cross-kingdom biofilms tested in this study could sufficiently lower the acidity of biofilm $(<\mathrm{pH} 4.5)$ that can cause enamel demineralization, regardless of the hyphal formation capability (Figure 4). Although we encountered a significant reduction in biofilm biomass when cultured with complete hyphal defective strain Ca_efg1 $\Delta \Delta$ in $\mathrm{SAL}_{100}$, interestingly, there were no significant differences in S. mutans population nor $\mathrm{pHs}$ of biofilm supernatant (Figures 4C, D). These results suggest that the hyphal transition of $C$. albicans induced by human saliva may facilitate the structural maturation of Sm-Ca biofilms (vs. SmCa_efg $1 \Delta \Delta$ ); the formation of small microcolonies along the hyphae during biofilm development might contribute to enhanced biofilm thickness of Sm-Ca biofilms (Supplementary Figure S6). Nevertheless, the alliance between $S$. mutans and yeast form of $C$. albicans in Sm-Ca_efg1 $1 \Delta$ was sufficient to surge their acidification. Since $S$. mutans binds to mannan in $C$. albicans cell wall via surface-formed glucans (Hwang et al., 2017), their symbiotic interaction could be less affected by the hyphal formation of $C$. albicans.

In addition, a previous study on metabolites/chromatographic analyses of bacterial-fungal derived conditioned medium revealed that it contained elevated amounts of organic acids; formate concentration was significantly increased in biofilms and $S$. mutans gtfBC expression was significantly enhanced which is essential for microcolony development (Kim et al., 2017). In our study, the accumulation of Sm-Ca biofilms on human enamel slab resulted in its erosion and severe alteration of surface topographies (Figure 5D). Thus, it is conceivable that Sm-Ca biofilms in saliva upregulated acid production (vs. Sm) thanks to synergistic interactions, thereby accelerating tooth decay. Therefore, it is necessary to investigate the metabolite composition in each biofilm cultured in saliva and analyze gene expressions associated with their metabolic activity during biofilm culturing to further understand the mechanism of action. Additionally, further studies regarding the role of salivary components on cross-kingdom interaction are required to fully understand these phenomena.

In summary, by performing in vitro experiments under more clinically relevant conditions (utilizing clarified human saliva), we provide feasible data to support the clinical findings in regards to the synergistic association between $S$. mutans and $C$. albicans in the pathogenesis of ECC. A key finding of this study is that the presence of $C$. albicans is critical to enhancing the maturity of biofilm and sustaining an acidic environment with $S$. mutans conducive to enamel demineralization in the $100 \%$ clarified saliva. While the hyphal transition of C. albicans assists further development of biofilm thickness, it is not essential for the acidogenicity of cross-kingdom biofilms. Our 
data explain, at least partly, why these organisms and rampant carious lesions are frequently observed from the plaque and teeth of children afflicted with ECC. Further understanding of the molecular biology mechanisms for S. mutans-C. albicans crosstalk in human saliva may lead to novel therapeutics to prevent and disrupt costly dental caries, such as ECC.

\section{DATA AVAILABILITY STATEMENT}

The original contributions presented in the study are included in the article/supplementary material. Further inquiries can be directed to the corresponding author.

\section{ETHICS STATEMENT}

The studies involving human participants were reviewed and approved by the Institutional Review Board of the University of Pennsylvania (protocol \#818549). The patients/participants provided their written informed consent to participate in this study.

\section{REFERENCES}

Ahn, S.-J., Ahn, S.-J., Wen, Z. T., Brady, L. J., and Burne, R. A. (2008). Characteristics of biofilm formation by Streptococcus mutans in the presence of saliva. Infect. Immun. 76, 4259-4268. doi: 10.1128/IAI.00422-08

Baillie, G. S., and Douglas, L. J. (1999). Role of dimorphism in the development of Candida albicans biofilms. J. Med. Microbiol. 48, 671-679. doi: 10.1099/ 00222615-48-7-671

Carlisle, P. L., and Kadosh, D. (2013). A genome-wide transcriptional analysis of morphology determination in Candida albicans. Mol. Biol. Cell 24, 246-260. doi: $10.1091 / \mathrm{mbc} . e 12-01-0065$

Cheaib, Z., Rakmathulina, E., Lussi, A., and Eick, S. (2015). Impact of acquired pellicle modification on adhesion of early colonizers. Caries Res. 49, 626-632. doi: 10.1159/000442169

Cocco, A. R., Cuevas-Suárez, C. E., Liu, Y., Lund, R. G., Piva, E., and Hwang, G. (2020). Anti-biofilm activity of a novel pit and fissure self-adhesive sealant modified with metallic monomers. Biofouling 36, 245-255. doi: 10.1080/ 08927014.2020.1748603

Colombo, N. H., Ribas, L. F., Pereira, J. A., Kreling, P. F., Kressirer, C. A., Tanner, A. C., et al. (2016). Antimicrobial peptides in saliva of children with severe early childhood caries. Arch. Oral. Biol. 69, 40-46. doi: 10.1016/j.archoralbio.2016.05.009

Cross, B. W., and Ruhl, S. (2018). Glycan recognition at the saliva-oral microbiome interface. Cell. Immunol. 333, 19-33. doi: 10.1016/ j.cellimm.2018.08.008

Dale, B. A., and Fredericks, L. P. (2005). Antimicrobial peptides in the oral environment: expression and function in health and disease. Curr. Issues Mol. Biol. 7, 119-133. doi: 10.1093/jac/dki103

Dawes, C., and Wong, D. (2019). Role of saliva and salivary diagnostics in the advancement of oral health. J. Dental Res. 98, 133-141. doi: 10.1177/ 0022034518816961

de Carvalho, F. G., Silva, D. S., Hebling, J., Spolidorio, L. C., and Spolidorio, D. M. P. (2006). Presence of mutans streptococci and Candida spp. in dental plaque/dentine of carious teeth and early childhood caries. Arch. Oral. Biol. 51, 1024-1028. doi: 10.1016/j.archoralbio.2006.06.001

Falsetta, M. L., Klein, M. I., Colonne, P. M., Scott-Anne, K., Gregoire, S., Pai, C.-H., et al. (2014). Symbiotic relationship between Streptococcus mutans and Candida albicans synergizes virulence of plaque biofilms in vivo. Infect. Immun. 82, 1968-1981. doi: 10.1128/IAI.00087-14

\section{AUTHOR CONTRIBUTIONS}

GH planned and constructed the study. H-EK, YL, AD, MB, $\mathrm{HK}$, and $\mathrm{GH}$ contributed to data acquisition and interpretation. $\mathrm{H}$-EK and $\mathrm{AD}$ performed all statistical analyses. H-EK, YL, AD, and GH drafted the manuscript and critically revised the manuscript. All authors contributed to the article and approved the submitted version.

\section{FUNDING}

This work was supported in part by the National Institutes for Dental and Craniofacial Research (NIDCR) grants DE027970 (GH) and DE025220 (HK).

\section{SUPPLEMENTARY MATERIAL}

The Supplementary Material for this article can be found online at: https://www.frontiersin.org/articles/10.3389/fcimb.2020. 623980/full\#supplementary-material

Gregoire, S., Xiao, J., Silva, B., Gonzalez, I., Agidi, P., Klein, M., et al. (2011). Role of glucosyltransferase B in interactions of Candida albicans with Streptococcus mutans and with an experimental pellicle on hydroxyapatite surfaces. Appl. Environ. Microbiol. 77, 6357-6367. doi: 10.1128/AEM.05203-11

Hajishengallis, E., Parsaei, Y., Klein, M. I., and Koo, H. (2017). Advances in the microbial etiology and pathogenesis of early childhood caries. Mol. Oral. Microbiol. 32, 24-34. doi: 10.1111/omi.12152

Hallett, K. B., and O'Rourke, P. K. (2002). Early childhood caries and infant feeding practice. Community Dental Health 19, 237-242.

Hara, A. T., and Zero, D. T. (2014). "The potential of saliva in protecting against dental erosion," in Erosive tooth wear (Basel, Switzerland: Karger Publishers), 197-205.

Helmerhorst, E. J., Hodgson, R., Van't Hof, W., Veerman, E., Allison, C., and Nieuw Amerongen, A. (1999). The effects of histatin-derived basic antimicrobial peptides on oral biofilms. J. Dental Res. 78, 1245-1250. doi: $10.1177 / 00220345990780060801$

Heydorn, A., Nielsen, A. T., Hentzer, M., Sternberg, C., Givskov, M., Ersbøll, BK., et al. (2000). Quantification of biofilm structures by the novel computer program COMSTAT. Microbiology 146 (10), 2395-2407.

Hwang, G., Marsh, G., Gao, L., Waugh, R., and Koo, H. (2015). Binding Force Dynamics of Streptococcus mutans-glucosyltransferase B to Candida albicans. J. Dental Res. 94, 1310-1317. doi: 10.1177/0022034515592859

Hwang, G., Liu, Y., Kim, D., Li, Y., Krysan, D. J., and Koo, H. (2017). Candida albicans mannans mediate Streptococcus mutans exoenzyme GtfB binding to modulate cross-kingdom biofilm development in vivo. PloS Pathog. 13, e1006407. doi: 10.1371/journal.ppat.1006407

ISO (2012). 25178-2: 2012-Geometrical Product Specifications (GPS)-Surface Texture: Areal-Part 2: Terms, Definitions and Surface Texture Parameters (Geneva, Switzerland: International Standards Organization).

Jurczak, A., Kościelniak, D., Papież, M., Vyhouskaya, P., and Krzyściak, W. (2015). A study on $\beta$-defensin- 2 and histatin-5 as a diagnostic marker of early childhood caries progression. Biol. Res. 48, 1-9. doi: 10.1186/s40659-0150050-7

Kadosh, D. (2013). Shaping up for battle: morphological control mechanisms in human fungal pathogens. PloS Pathog. 9, e1003795. doi: 10.1371/ journal.ppat.1003795

Kim, D., Liu, Y., Benhamou, R. I., Sanchez, -H., Simón-Soro, A., Li, Y., et al. (2018). Bacterial-derived exopolysaccharides enhance antifungal drug 
tolerance in a cross-kingdom oral biofilm. ISME J. 12(6), 1427-1442. doi: $10.1038 /$ srep41332

Kim, D., Sengupta, A., Niepa, T. H., Lee, B.-H., Weljie, A., Freitas-Blanco, V. S., et al. (2017). Candida albicans stimulates Streptococcus mutans microcolony development via cross-kingdom biofilm-derived metabolites. Sci. Rep. 7, 1-14. doi: $10.1038 /$ srep 41332

Klein, M. I., Scott-Anne, K. M., Gregoire, S., Rosalen, P. L., and Koo, H. (2012). Molecular approaches for viable bacterial population and transcriptional analyses in a rodent model of dental caries. Mol. Oral. Microbiol. 27, 350361. doi: 10.1111/j.2041-1014.2012.00647.x

Koo, H., Xiao, J., Klein, M., and Jeon, J. (2010). Exopolysaccharides produced by Streptococcus mutans glucosyltransferases modulate the establishment of microcolonies within multispecies biofilms. J. Bacteriol. 192, 3024-3032. doi: 10.1128/JB.01649-09

Larsen, M., and Pearce, I. (1997). A computer program for correlating dental plaque $\mathrm{pH}$ values, $\mathrm{cH}+$, plaque titration, critical $\mathrm{pH}$, resting $\mathrm{pH}$ and the solubility of enamel apatite. Arch. Oral. Biol. 42, 475-480. doi: 10.1016/ S0003-9969(97)00044-7

Liu, H., Kohler, J., and Fink, G. R. (1994). Suppression of hyphal formation in Candida albicans by mutation of a STE12 homolog. Science 266, 1723-1726. doi: $10.1126 /$ science.7992058

Liu, Y., Naha, P. C., Hwang, G., Kim, D., Huang, Y., Simon-Soro, A., et al. (2018). Topical ferumoxytol nanoparticles disrupt biofilms and prevent tooth decay in vivo via intrinsic catalytic activity. Nat. Commun. 9, 1-12. doi: 10.1038/s41467018-05342-x

Marsh, P. D. (2010). Microbiology of dental plaque biofilms and their role in oral health and caries. Dental Clinics 54, 441-454. doi: 10.1016/ j.cden.2010.03.002

Murakami, S., Mealey, B. L., Mariotti, A., and Chapple, I. L. (2018). Dental plaqueinduced gingival conditions. J. Clin. Periodontol. 45, S17-S27. doi: 10.1002/ JPER.17-0095

Murray, P., Prakobphol, A., Lee, T., Hoover, C., and Fisher, S. (1992). Adherence of oral streptococci to salivary glycoproteins. Infect. Immun. 60, 31-38. doi: 10.1128/IAI.60.1.31-38.1992

O'Sullivan, J. M., Jenkinson, H. F., and Cannon, R. D. (2000). Adhesion of Candida albicans to oral streptococci is promoted by selective adsorption of salivary proteins to the streptococcal cell surface. Microbiology 146, 41-48. doi: 10.1099/00221287-146-1-41

Parisotto, T. M., Steiner-Oliveira, C., Silva, C. M. S. E., Rodrigues, L. K. A., and Nobre-Dos-Santos, M. (2010). Early childhood caries and mutans streptococci: a systematic review. Oral. Health Prev. Dent. 8, 59-70. doi: 10.3290/ j.ohpd.a18828

Paula, A. J., Hwang, G., and Koo, H. (2020). Dynamics of bacterial population growth in biofilms resemble spatial and structural aspects of urbanization. Nat. Commun. 11, 1-14. doi: 10.1038/s41467-020-15165-4
Pedersen, A. M. L., and Belstrøm, D. (2019). The role of natural salivary defences in maintaining a healthy oral microbiota. J. Dent. 80, S3-S12. doi: 10.1016/ j.jdent.2018.08.010

Pereira, D., Seneviratne, C., Koga-Ito, C., and Samaranayake, L. (2018). Is the oral fungal pathogen Candida albicans a cariogen? Oral. Dis. 24, 518-526. doi: 10.1111/odi.12691

Phattarataratip, E., Olson, B., Broffitt, B., Qian, F., Brogden, K. A., Drake, D. R., et al. (2011). Streptococcus mutans strains recovered from caries-active or caries-free individuals differ in sensitivity to host antimicrobial peptides. Mol. Oral. Microbiol. 26, 187-199. doi: 10.1111/j.2041-1014.2011.00607.x

van't Hof, W., Veerman, E. C., Amerongen, A. V. N., and Ligtenberg, A. J. (2014). "Antimicrobial defense systems in saliva," in Saliva: Secretion and functions (Basel, Switzerland: Karger Publishers), 40-51.

Vorregaard, M. (2018). Comstat2-a modern 3D image analysis environment for biofilms. Master's thesis, Master's thesis. Lyngby, Denmark: Technical University of Denmark, DTU, DK-2800 Kgs.

Xiao, J., Klein, M. I., Falsetta, M. L., Lu, B., Delahunty, C. M., Yates Iii, J. R., et al. (2012). The exopolysaccharide matrix modulates the interaction between 3D architecture and virulence of a mixed-species oral biofilm. PloS Pathog. 8, e1002623. doi: 10.1371/journal.ppat.1002623

Xiao, J., Moon, Y., Li, L., Rustchenko, E., Wakabayashi, H., Zhao, X., et al. (2016). Candida albicans carriage in children with severe early childhood caries (SECC) and maternal relatedness. PloS One 11, e0164242. doi: 10.1371/ journal.pone. 0164242

Xiao, J., Hara, A. T., Kim, D., Zero, D. T., Koo, H., and Hwang, G. (2017). Biofilm three-dimensional architecture influences in situ $\mathrm{pH}$ distribution pattern on the human enamel surface. Int. J. Oral. Sci. 9, 74-79. doi: 10.1038/ijos.2017.8

Xiao, J., Huang, X., Alkhers, N., Alzamil, H., Alzoubi, S., Wu, T. T., et al. (2018). Candida albicans and early childhood caries: a systematic review and metaanalysis. Caries Res. 52, 102-112. doi: 10.1159/000481833

Yang, X. Q., Zhang, Q., Lu, L. Y., Yang, R., Liu, Y., and Zou, J. (2012). Genotypic distribution of Candida albicans in dental biofilm of Chinese children associated with severe early childhood caries. Arch. Oral. Biol. 57, 10481053. doi: 10.1016/j.archoralbio.2012.05.012

Conflict of Interest: The authors declare that the research was conducted in the absence of any commercial or financial relationships that could be construed as a potential conflict of interest.

Copyright $\odot 2021 \mathrm{Kim}, \mathrm{Liu}$, Dhall, Bawazir, Koo and Hwang. This is an open-access article distributed under the terms of the Creative Commons Attribution License (CC BY). The use, distribution or reproduction in other forums is permitted, provided the original author(s) and the copyright owner(s) are credited and that the original publication in this journal is cited, in accordance with accepted academic practice. No use, distribution or reproduction is permitted which does not comply with these terms. 